OppA (oligopeptide binding protein A), the periplasmic component of another uptake transporter, appears to cooperate with the Sap transporter for LL37 uptake. For beta-defensin resistance, H. ducreyi utilises the MTR efflux transporter. MTR is a member of the resistance-nodulation-division family of multidrug resistance transporters that pump hydrophobic agents from the periplasm and cytoplasm out of the cell. Our data demonstrate that the H. ducreyi MTR transporter confers resistance to both LL37 and beta-defensins. Interestingly, we also found that the MTR transporter affects activation of CpxRA, which globally regulates virulence factors in $\mathrm{H}$. ducreyi. The role of MTR in human virulence is under investigation. Together, these studies highlight the significance of AP resistance mechanisms to pathogen survival in the human host.

\section{S07.4 IDENTIFICATION OF DETERMINANTS TRIGGERING ANTIGENIC VARIATION IN MYCOPLASMA GENITALIUM}

doi:10.1136/sextrans-2013-051184.0041

R Burgos, P A Totten. University of Washington, Seattle, WA, United States

Mycoplasma genitalium (MG) is an emerging sexually transmitted pathogen associated with reproductive tract disease in men and women. Despite the development of a robust antibody response, MG can persist for months to years, potentially increasing the risk for sexual transmission and serious upper reproductive tract infection in women. The molecular basis of MG pathogenesis is poorly understood, in part due to its fastidious nature, extremely small genome lacking known virulence genes, and the limited genetic tools available for molecular investigations. Nevertheless, previous studies have linked MG virulence to its unique terminal organelle, a complex structure that mediates adherence, motility, and cell division. The terminal organelle is composed of a complex array of unique proteins, including $\mathrm{MgpB}$ and $\mathrm{MgpC}$ which serve as major adhesins and are required for terminal organelle biogenesis. Remarkably, these two surface-exposed proteins also undergo phase and antigenic variation through a unique process of segmental recombination between discreet variable regions within $m g p B$ and $m g p C$ and multiple homologous archived sequences, termed $\mathrm{MgPa}$ repeats (MgPar). Our goal is to identify the molecular factors required to promote this genetic diversity, a mechanism which likely contributes to the ability of MG to adapt to different host conditions and maintain persistent infections. Recently, we have shown that RecA is required for $m g p \mathrm{~B} / \mathrm{C}$ gene variation and that this protein is expressed in several isoforms. We have now expanded these studies by showing that these RecA isoforms originate from different translational start sites and that specific rec $\mathrm{A}$ upstream sequences regulate the expression ratio of these isoforms and $m g p B / C-M g P a r$ recombination. Together, these studies suggest the presence of novel regulatory mechanisms that may allow this genetically challenged organism to cause disease, evade the host immune response, and persist in infected individuals.

\section{SO7.5 UNDERSTANDING DISSEMINATION OF TREPONEMA PALLIDUM WITHIN THE HOST - IS THERE HOPE FOR A SYPHILIS VACCINE?}

doi:10.1136/sextrans-2013-051184.0042

S Houston, R Hof, C Wetherell, A Phillips, Y Denchev, C E Cameron. Department of Biochemistry and Microbiology, University of Victoria, Victoria, BC, Canada

Treponema pallidum is a highly invasive spirochete that disseminates to organ sites distal to the site of primary infection and is able to cross both the blood-brain and placental barriers during the course of infection. The corkscrew motility used by T. pallidum undoubtedly contributes to its invasive nature. However, this signature motility is shared with other spirochetes and thus the factors responsible for the widespread dissemination capability that is unique to $T$. pallidum remain unknown. We have identified the treponemal-specific, surface-localised protein pallilysin as a dual functioning adhesin/metalloprotease that exhibits specific attachment to, and degradation of, multiple extracellular matrix components. Pallilysin is produced as an inactive proprotease that can be activated via either autocatalytic cleavage or host-originating thrombin cleavage. Purified recombinant pallilysin, as well as a noninvasive model treponeme heterologously expressing pallilysin on its surface, exhibit specific degradation of fibrin clots. Pallilysin immunisation alters the course of T. pallidum dissemination following challenge within the rabbit model of syphilis infection, with immunised rabbits exhibiting a reduced bacterial burden within organs distal to the site of challenge compared to unimmunized control rabbits. Further, rabbit infectivity tests (RIT) showed that rabbits receiving lymph nodes from challenged, unimmunized rabbits seroconverted and developed orchitis by 30 days post-transfer, while $66 \%$ of RIT rabbits receiving lymph nodes from challenged, pallilysin-immunised rabbits remained seronegative and had no signs of orchitis at the termination of the experiment (day 185 posttransfer). Collectively these studies identify pallilysin as a $T$. pallidum-specific metalloprotease that (1) exploits the host coagulation cascade to facilitate protease activation, (2) plays a central role in treponemal dissemination and (3) shows promise as a novel syphilis vaccine candidate.

\section{S.08 - STI/HIV treatment guidelines: Important areas of clinical uncertainty}

\section{S08.1 TREATMENT OF GONORRHOEA IN AN ERA OF EMERGING CEPHALOSPORIN RESISTANCE AND RESULTS OF A RANDOMISED TRIAL OF NEW POTENTIAL TREATMENT OPTIONS}

doi:10.1136/sextrans-2013-051184.0043

R D Kirkcaldy. CDC, Atlanta, GA, United States

Background Cephalosporins are the mainstay of recommended gonorrhoea treatment, and there is an urgent need for treatment options for cephalosporin-allergic patients or infections with suspected cephalosporin resistance. We evaluated the efficacy and tolerability of two novel combinations of existing antimicrobials for treatment of uncomplicated urogenital gonorrhoea.

Methods We conducted a non-comparative trial, randomising patients with urogenital gonorrhoea to one of two regimens: gentamicin $240 \mathrm{mg}$ intramuscularly plus azithromycin $2 \mathrm{~g}$ orally (GENT/ AZI), or gemifloxacin $320 \mathrm{mg}$ orally plus azithromycin $2 \mathrm{~g}$ orally (GEMI/AZI). The primary outcome was microbiologic cure of urogenital infections (negative follow-up culture) at 10-17 days posttreatment. All participants who returned for follow-up and had evaluable follow-up cultures were included in this per protocol analysis.

Results For 401 evaluable participants (GENT/AZI-202; GEMI/ AZI-199), the mean age of both groups was 30 years. Most participants were heterosexual men (GENT/AZI, 57\%; GEMI/AZI, 51\%), followed by men who have sex with men (GENT/AZI, 33\%; GEMI/ AZI, 39\%), and women (GENT/AZI, 9\%; GEMI/AZI, 11\%). Microbiological cure was achieved by 100\% (lower one-sided $95 \%$ confidence interval, 98.5\%) of GENT/AZI participants, and 99.5\% (lower one-sided $95 \%$ confidence interval, $97.6 \%$ ) of GEMI/AZI participants. GENT/AZI cured 10/10 pharyngeal and $1 / 1$ rectal infections; GEMI/AZI cured $15 / 15$ pharyngeal and $5 / 5$ rectal infections. In the GENT/AZI arm, the most common adverse events (AEs) were mildmoderate nausea (27\% of participants), diarrhoea (19\%), abdominal discomfort/pain and vomiting (both 7\%). In the GEMI/AZI arm, the most common AEs were nausea (37\% [8\% moderate-severe]), diarrhoea (23\%), and abdominal discomfort/pain (11\%). 